\section{Abfallwirtschaftspreis}

\author{
„Phönix“ 2008 und Sonderpreise ausgeschrieben
}

esucht werden kreative, praxistaugliche und/oder innovative Lösungen und Konzepte, die zu einer nachhaltigen Entwicklung der Abfallwirtschaft beitragen.

Die Einreichungen unterliegen keiner thematischen Einschränkung, sie können z. B. aus den Gebieten der Technik, der Wissenschaft, der abfallwirtschaftlichen Praxis, aber auch der Öffentlichkeitsarbeit stammen. Zentrale Beurteilungskriterien sind Praxistauglichkeit, Nachhaltigkeit und abfallwirtschaftliche Relevanz. Der Hauptpreis ist mit 8.000 Euro Preisgeld dotiert, aus Anlass des 10-jährigen
„Phönix“-Jubiläums werden zusätzlich ein Sonderpreis für Kommunen und ein Sonderpreis Vermeidung des ARA-Systems (je 2.000 Euro) vergeben.

Einreichschluss: 31. März 2008

Die Preisverleihung erfolgt am 15. Mai 2008 in Wien.

Der „Phönix“ ist eine Initiative des Österreichischen Wasser- und Abfallwirtschaftsverbands (ÖWAV) und des Lebensministeriums.

Einreichunterlagen und weitere Informationen: ÖWAV, Mag. Fritz Randl, 1010 Wien, Marc-AurelStraße 5, Telefon 01/535 57 20, randl@oewav.at, www.oewav.at 〉> Phönix 2008

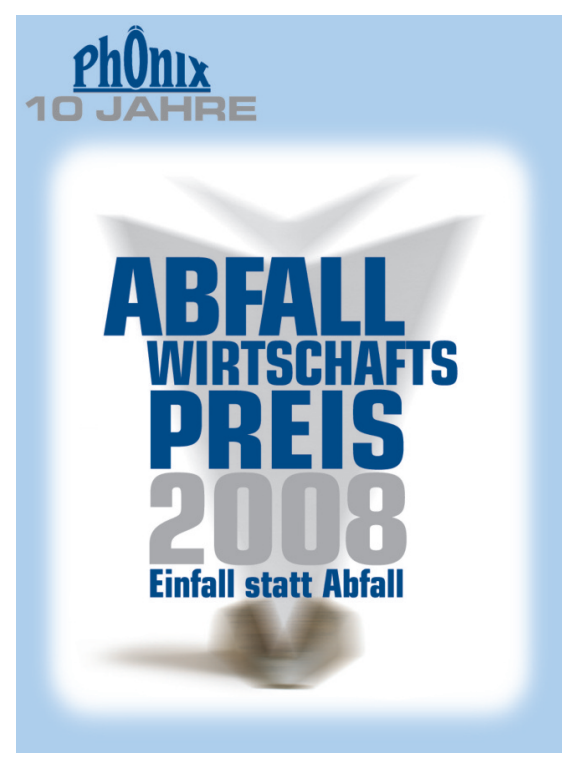

\title{
Aus- und Weiterbildung
}

WKO campus wien eröffnet

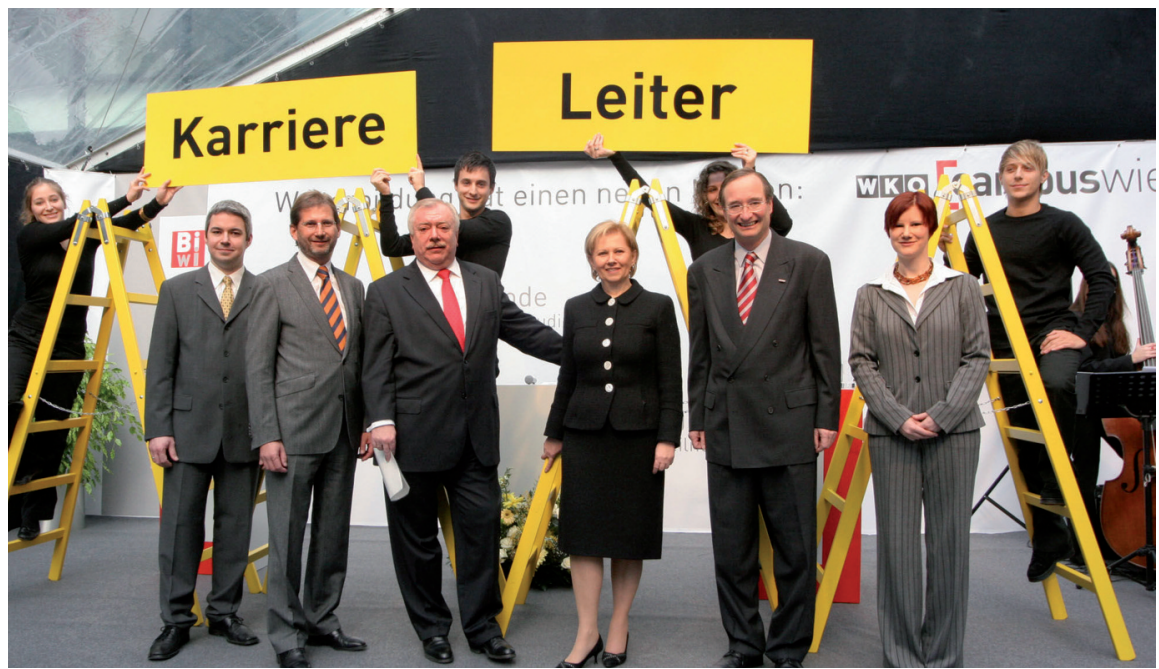

Eröffnung des neuen WKO-Campus durch Bgm. Dr. Michael Häupl, BM Dr. Johannes Hahn, WKW-Präs. KR Brigitte Jank und WKO-Präs. Dr. Christoph Leitl (Foto: Pressefoto Votava)

(RK) Als wichtige Investition in die $\mathrm{Zu}$ kunft der Stadt Wien als mitteleuropäische Wissensmetropole bezeichnete Bürgermeister Dr. Michael Häupl den „WKO campus wien“ am Währinger Gürtel, der am 5. Dezember 2007 eröffnet wurde. Gemeinsam mit der Präsidentin der Wirtschaftskammer Wien, KommR Brigitte Jank, Wissenschaftsminister Dr. Johannes Hahn und WKO-Präsident Dr. Christoph Leitl würdigte der Wiener Bürgermeister die Bildungseinrichtung als kräftigen Impuls für den Wissens- und Wirtschaftsstandort Wien. Der neue WKO campus wien, der durch den Um- und Ausbau der bereits beste- henden Bildungseinrichtungen der Kammer an diesem Standort hervorgegangen ist, bietet nun auf 63.000 Quadratmetern in sechs Etagen ein noch nie dagewesenes Angebot an Seminaren, Kursen und Lehrgängen unter einem Dach. Zahlreiche Persönlichkeiten aus Politik, Wirtschaft und aus dem Bildungsbereich nahmen an der Eröffnungsfeier teil.

Aus- und Weiterbildung seien im internationalen Wettbewerb wesentliche Elemente für die Erhaltung der Konkurrenzfähigkeit und damit des Wohlstandes auch in kommenden Jahren. Wien sei als Bildungsstandort mit der Qualität und der Bandbreite der entsprechenden Einrichtungen, wie auch mit der Vernetzung derselben gut aufgestellt. Es gelte jedoch, permanent an derVerbesserung aller Standards zu arbeiten. Der unkomplizierte Zugang und die hohe Praxisorientierung mache die spezielle Qualität der Bildungseinrichtungen der Wirtschaftskammer aus, die vor allem der kleinteiligen Wiener Wirtschaft zugute komme.

Mit dem Bau seien ideale Bedingungen für die hier Studierenden geschaffen worden. Die Kammer habe damit in ihrer langen Tradition der Ausbildung gehandelt und eine Einrichtung geschaffen, die letztlich der Qualität und den Leistungen der österreichischen Wirtschaft und damit der Konkurrenzfähigkeit in der globalisierten Welt zugute komme.

Der WKO campus wien vereint nun mit dem Bildungs- und Beratungsangebot des WIFI Wien, allen acht FHWienStudiengängen der WKW, der Werbe Akademie und dem Berufsinformationszentrum der Wiener Wirtschaft eine ganze Reihe von Bildungseinrichtungen unter einem Dach. Das Angebot für die Studierenden konnte damit in allen Bereichen ausgeweitet werden, allein die nunmehr am Währinger Gürtel vereinten acht FHWien-Studiengänge bieten Raum für 2.400 Studenten.

Der Um- und Neubau wurde in 18 Monaten bewerkstelligt, die Kosten des auch architektonisch ansprechenden Baues betrugen 27,4 Millionen Euro.

Weitere Informationen:

www.wifiwien.at, www.fh-wien.ac.at, www.werbeakademie.at bzw. www.biwi.at 


\section{Mülltrennung}

Sammelmoral in Österreich ungebrochen

(OTS) Alle Jahre wieder beschert das Weihnachtsgeschäft der österreichischen Wirtschaft Rekordumsätze: 2008 haben die Österreicher rund 1,6 Milliarden Euro für Weihnachtsgeschenke ausgegeben. Mit dem Konsum steigen aber nicht nur die Umsätze, sondern auch die Abfallmengen, und die Entsorger verzeichnen zu den Festtagen immer neue Rekorde. Erfreulich ist daher, dass für die Österreicher umweltgerechtes Verhalten und die getrennte Sammlung außer Frage stehen.

Die Österreicher nehmen die Schonung der natürlichen Ressourcen ernst: Bei einer repräsentativen Umfrage der Karmasin Marktforschung (1.000 Befragte) im Auftrag des ARA Systems bezeichneten 90 Prozent der Befragten die Sammlung wieder verwertbarer Stoffe als sinnvoll.

Ist das nur ein Lippenbekenntnis? Die Kontrollfrage zeigt, dass die Österreicher das Recycling von Wertstoffen und ihren persönlichen Beitrag dazu sehr ernst nehmen: 95 Prozent (regelmäßig: 73 Prozent, ab und zu: 22 Pro- zent) sammeln zu Hause Verpackungen getrennt vom sonstigen Restmüll und bringen Dosen, Kunststoffflaschen, Altpapier und Glas zu den Sammelbehältern. Sechs von zehn Österreichern sehen darin im Alltag nicht einmal mehr einen zusätzlichen Zeitaufwand.

Denn die Österreicher haben sich an die getrennte Wertstoffsammlung längst als Teil ihres täglichen Lebens gewöhnt: 76 Prozent könnten sich laut Umfrage nicht einmal mehr vorstellen, auf die getrennte Sammlung zu verzichten. Glas, Dosen oder Altpapier achtlos in den Restmüll zu werfen, ist also „out“.

Zwei Gründe für die hohe Sammelbereitschaft sind die flächendeckende Verfügbarkeit von Sammelbehältern sowie das hohe Wissen um den Wert dieser wieder verwertbaren Materialien für die Schonung der natürlichen Lebensressourcen: Acht von zehn Befragten betonen, dass eine saubere Entsorgung von Wertstoffen auch für den Tourismus und die Verschönerung des Orts- oder Stadtbildes wichtig sei. Das ARA System sieht diesen erfreulichen Umstand als Ergeb-

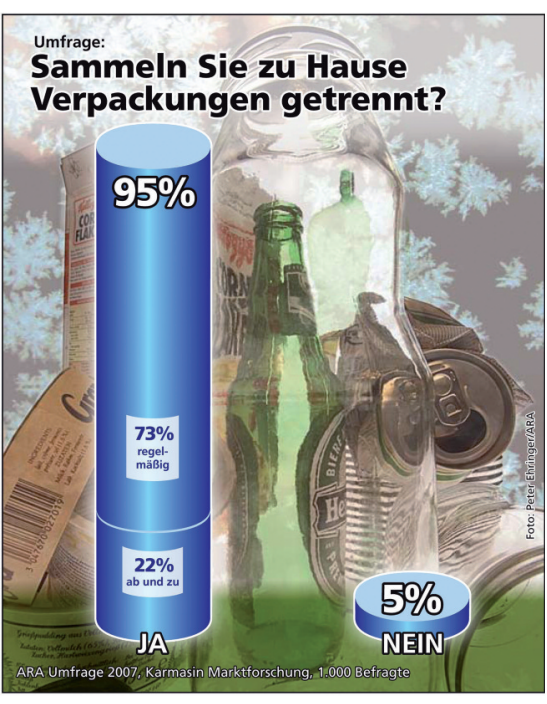

95 Prozent der Österreicher tragen Dosen, Kunststofffllaschen, Altpapier und Glas zu

95 Prozent der Österreicher bringen Dosen, Kunststoffflaschen, Altpapier und Glas zu den dafür aufgestellten Sammelbehältern

nis der gemeinsamen Arbeit von Kommunen, Wirtschaft und ARA System. Besonders wichtig sei es, den Bürgern nicht nur zu erklären, wie gesammelt werden muss, sondern auch warum. Denn wer den Sinn der getrennten Sammlung erkennt, ist viel eher bereit, seinen Beitrag zu leisten.

\section{Forschungs- förderung}

FFG-Brückenschlagprogramm (BRIDGE) - Ausschreibung 2008

(FFG) BRIDGE, das FFG-Brückenschlagprogramm, wurde als Förderinstrument für Projekte zwischen Grundlagenforschung und angewandter Entwicklung eingerichtet. Gefördert werden Kooperationsprojekte zwischen wissenschaftlichen Instituten und Unternehmen mit überwiegendem Grundlagenforschungscharakter, die jedoch bereits ein realistisches Verwertungspotenzial erkennen lassen. Die Förderung bezieht sich auf Einzelprojekte, und ist für alle Forschungsthemen offen.

Das Brückenschlagprogramm hat Anfang Dezember 2007 die nunmehr 7. Ausschreibung gestartet. Einreichungen sind bis 27. März 2008, 12.00 Uhr, ausschließlich per eCall möglich.

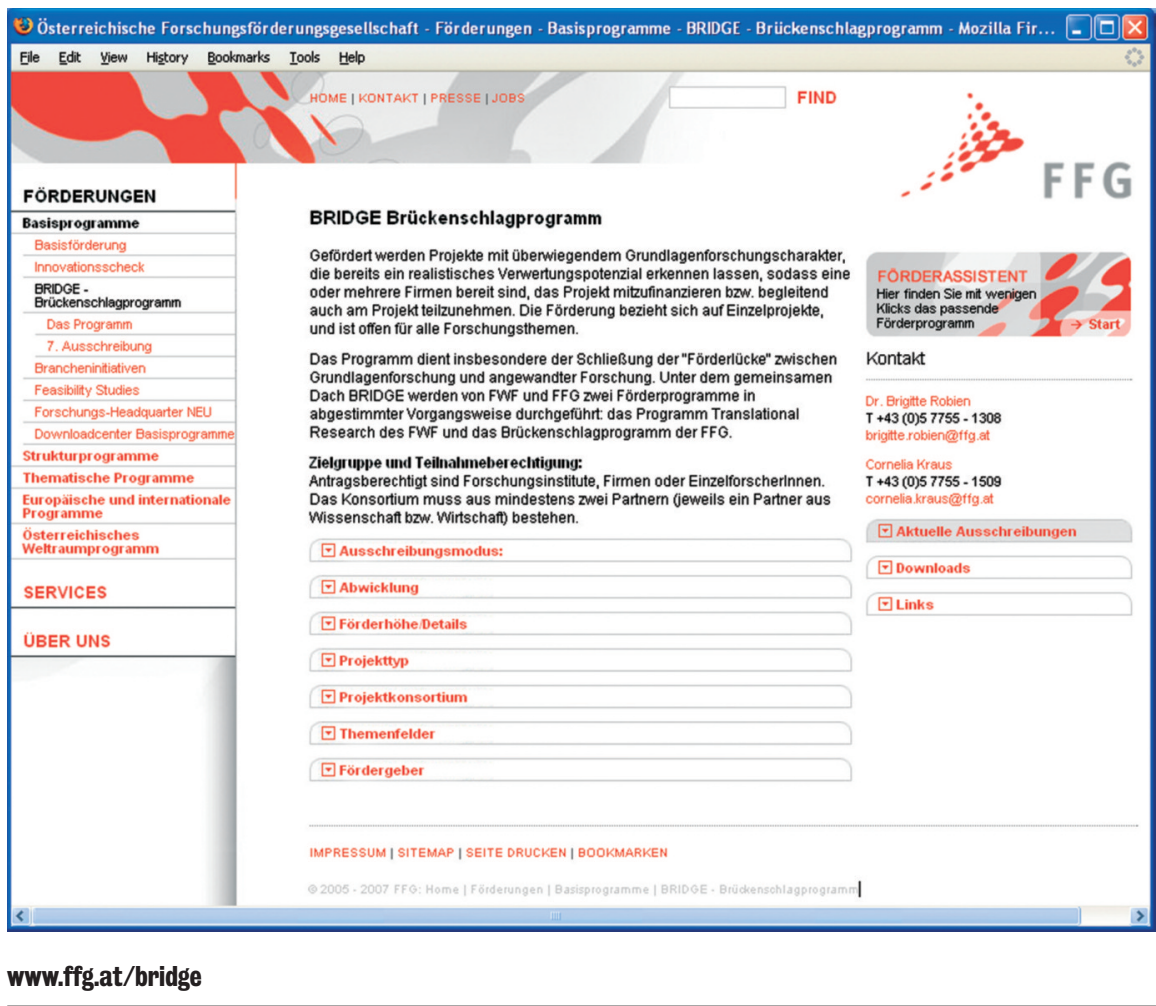




\section{Hochwasservorsorge}

Zusätzliche Schutzmaßnahmen haben sich bewährt

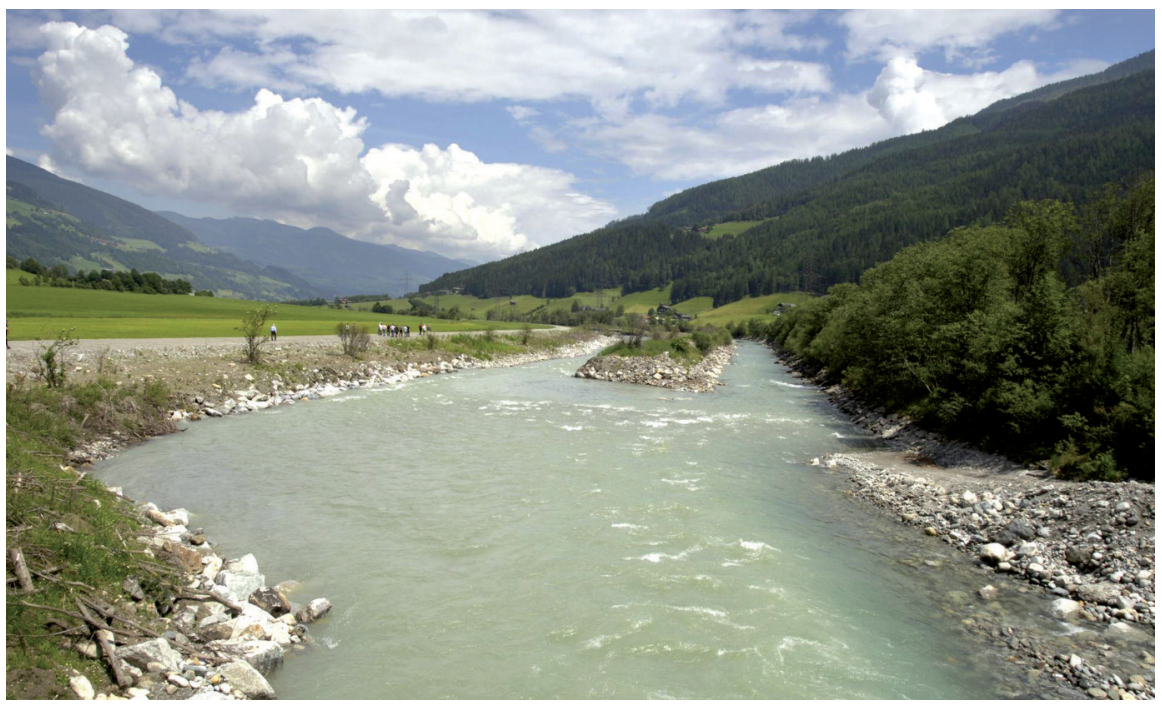

Hochwasserschutz Salzach/Bramberg (Foto: BMLFUW/Rita Newman)

(BMLFUW) Die Analysen der Hochwasserereignisse der letzten Jahre haben die strategischen Zielsetzungen des Lebensministeriums, die sich aus der Hochwasserkatastrophe 2002 ergeben haben, bestätigt. Dort, wo die Niederschläge innerhalb eines durchschnittlichen hundertjährigen Ereignisses geblieben sind, haben sich die Schutzmaßnahmen bewährt. Das heißt, dass durch zusätzliche präventive Maßnahmen die Auswirkungen der Hochwasserereignisse in Österreich seit 2002 verringert werden konnten.

Die kompetenzübergreifende Koordination der Maßnahmen zwischen allen Gebietskörperschaften und den involvierten Organisationen hat sich darüber hinaus als einziger Weg herausgestellt, Katastrophen wie etwa im Jahr 2002 zu bewältigen.

Große Schäden nach Hochwasser sind in den vergangen Jahren primär dort aufgetreten, wo ein durchschnittliches hundertiähriges Ereignis weit überschritten wurde. Nach wie vor stellen Hangmuren an zahlreichen Stellen eine versteckte Gefahr dar, die mit technischen Schutzmaßnahmen alleine kaum bewältigt werden kann. Die Berücksichtigung von Hangprozessen in der Gefahrenzonenplanung ist somit eine große Herausforderung für die Sicherheit in der Raumordnung und im Bauwesen.

Auch im neuen Programm der Ländlichen Entwicklung 2007-2013 stellt das
Thema „Naturgefahren“ einen Schwerpunkt dar. Pro Jahr sind nun rund 17,5 Millionen Euro für Maßnahmen zum Schutz vor Naturgefahren vorhanden. Weiters stellt der nationale Katastrophenfonds seit 2007 zusätzlich 37 Millionen Euro jährlich zur Verfügung.

Ein erhebliches Problem ist jedoch nach wie vor die starke Zunahme von Schäden an Gebäuden oder Inventar in den Gebieten mit Restgefährdung durch Hochwasser, Wildbäche und Muren. Das verbleibende Restrisiko wird daher künftig auch bei allen Planungen von neuen Maßnahmen verstärkt berücksichtigt werden. Auch Eigenvorsorge und Gebäudeschutz sind ein Gebot der Stunde. Das Lebensministerium setzt dabei intensiv auf Risikokommunikation und Bürgerbeteiligung - insbesondere bei der Erstellung der Gefahrenzonenpläne und der Umsetzung von Schutzprojekten.

Das Lebensministerium beteiligte sich in der abgelaufenen Programmperiode federführend an insgesamt vier Projekten des INTERREG-Programms einer europäischen Raumplanungsinitiative, die aus Mitteln der EU-Strukturfonds finanziert wird. Dabei konnten für die Fragestellungen des Schutzes vor Naturgefahren mehr als 700.000 Euro an EU-Mitteln lukriert werden. Für das Nachfolgeprogramm „Europäische Territoriale Kooperation" laufen bereits Vorbereitungen zur Teilnahme an den Ausschreibungen der drei Programmsäulen. 


\section{AKTUELL}

\section{Abfallvermeidung}

ERP-Ideenwettbewerb bis 30 . April verlängert

(OTS) Die Europäische Recycling Plattform ERP verlängert die Einreichfrist für ihren Ideenwettbewerb bis 30. April 2008. Gefragt sind kreative Vorschläge, die den steigenden Müllbergen an ausgedienten Elektro- und Elektronikgeräten den Kampf ansagen. Die Ausschreibung richtet sich an junge ForscherInnen und EntwicklerInnen, die in Österreich tätig sind.

In ihren Beiträgen sollen sich die EinreicherInnen mit einer oder mehreren der folgenden Fragen beschäftigen:
- Welche Maßnahmen können bereits bei der Gestaltung von Elektro- und Elektronikgeräten umgesetzt werden, damit später weniger Abfall entsteht?

- Wie können neue Produktideen aussehen, die weniger Abfall erzeugen?

- Wie kann die Verpackung der Geräte zur Abfallvermeidung beitragen?

Alle eingesandten Vorschläge werden von einem ausgewählten, externen Expertengremium bewertet, das sich aus wissenschaftlichen Partnern des Kom-
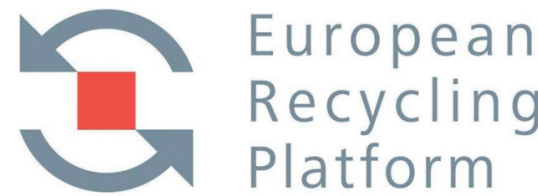

petenzzentrums Elektronik und Umwelt KERP und einem Vertreter des Lebensministeriums zusammensetzt.

Der Ideenwettbewerb wird im Auftrag der ERP von der KERP Consulting $\mathrm{GmbH}$ organisiert und durchgeführt.

Die Bekanntgabe der GewinnerInnen und die Preisverleihung finden im Rahmen einer öffentlichen Veranstaltung Ende Mai 2008 in Wien statt.

Teilnahmebedingungen und weitere

Informationen: www.kerp.at

\section{Normung}

\section{Normungsinstitut startet „Austrian Standards plus $\mathrm{GmbH}^{\text {“ }}$}

(pts) Mit wichtigen Neuerungen wartet das Österreichische Normungsinstitut (ON) zu Jahresbeginn 2008 auf: Die Geschäftsbereiche Verkauf \& Service sowie Seminare und Zertifizierung wird künftig die neu gegründete Austrian Standards plus $\mathrm{GmbH}$ (AS+) übernehmen. AS+ steht zu 100 Prozent im Eigentum des ON und gliedert sich in die Unternehmensbereiche

- Austrian Standards plus Publishing (bisher ON Sales \& Service),

- Austrian Standards plus Trainings (bisher ON Seminare) sowie

- Austrian Standards plus Certification (bisher ON Certification).

„Mit der Gründung der AS+ will das Nor-

\section{Austrian Standards plus More Than Just Standards. \\ Das Logo der Austrian Standards plus GmbH (Bild: ON)}

mungsinstitut in erster Linie seinen Kundenservice rund um Normen weiter verbessern“, so Direktor Dr. Gerhard Hartmann, Geschäftsführer des ON und der AS+. „Das Österreichische Normungsinstitut war und ist immer bemüht, seinen Kunden mehr zu bieten als Normen, nämlich mehr an Informationen, mehr an intelligenten und hochwertigen $\mathrm{Zu}$ satzprodukten und ein Plus an Weiterbildungs- und Zertifizierungsmöglichkeiten.“

Mit einer eigenen $\mathrm{GmbH}$, die diese Bereiche übernimmt, könne man nun rascher und individueller auf Kunden- bedürfnisse reagieren, den Kunden neue und innovative Produkte und Dienstleistungen professioneller anbieten bzw. diese auch mit ihnen entwickeln.

Für die Erstellung von Normen und Regelwerken bleibt weiterhin das Österreichische Normungsinstitut ON mit seinen Komitee-Managern die zuständige kompetente Plattform.

Mehr Informationen zur Austrian Standards plus $\mathrm{GmbH}$ sowie zu den unterschiedlichen Tätigkeitsbereichen und Angeboten gibt es unter www.as-plus.at sowie auf der Website des Normungsinstituts www.on-norm.at/publish/info asplus neu.html.

\section{Innverlegung}

Renaturierung der Mündungsbereiche von Zubringern verbessert Hochwasserschutz

(BMLFUW) Die Verlegung des Inns beim Innsbrucker Flughafen stellt nicht nur eine rein sicherheitstechnische Maßnahme dar - um den von der Internationen Zivilluftfahrt (ICAO) geforderten Sicherheitsstandard zu erfüllen -, sondern bringt auch umwelttechnisch gesehen eine ökologisch wertvolle Umgestaltung des Flusses sowie seiner Seitenzubringer. Neben der Renaturierung der Mün- dungsbereiche des Völser Gießen und des Axamer Baches werden der Inn und das Innufer im Baubereich ökologisch aufgewertet.

Das Lebensministerium investiert dafür Bundesmittel in der Höhe von rund 2,65 Millionen Euro. Mit Ergänzungsmaßnahmen kann in weiterer Folge auch ein verbesserter Hochwasserschutz für die Marktgemeinde Völs erreicht werden, so Umweltminister Pröll am 16. Jänner 2008 anlässlich des Spatenstichs zur Innverlegung am Innsbrucker Flughafen.

Die Internationale Zivilluftfahrtbehörde schreibt vor, dass jeder Flughafen nach dem Pistenende über ein 150 Meter langes Pistenvorfeld verfügen muss. Da am Flughafen Innsbruck am westlichen Pistenende nur 60 Meter zur 
Verfügung standen, wurde der Tiroler Flughafenbetriebsgesellschaft $\mathrm{mbH}$ (TFG) im Jahr 2005 durch das Bundesministerium für Verkehr, Innovation und Technologie die luftfahrtrechtliche Bewilligung zur Änderung der festgelegten Flughafengrenzen erteilt. Ziel war die Herstellung eines dem internationalen Standard entsprechenden Pistenvorfeldes am Flughafen Innsbruck.

Im September 2007 wurde weiters das wasser-, naturschutz- und forstrechtliche Verfahren für das Sicher- heitsprojekt „Innverlegung“ positiv abgewickelt. Bereits im Jänner 2008 wurde mit der Einrichtung der Baustelle auf Völser Seite begonnen. Die erste Bauphase wird im März/April dieses Jahres abgeschlossen, im Sommer ruht die Arbeit. Im Oktober/November 2008 werden die Bautätigkeiten wieder aufgenommen, um das Projekt fristgerecht bis zum Frühjahr 2009 fertig zu stellen. Durch die vorgesehenen Maßnahmen wird nun nicht nur das international geforderte Pistenvorfeld geschaffen, sondern auch der Inn ökologisch wertvoll umgestaltet werden. So entstehen unter anderem eine Insel und ein Seitenarm des Inns.

Die Baukosten belaufen sich insgesamt auf rund 8,26 Millionen Euro. In Summe werden rund $250.000 \mathrm{~m}^{3}$ Material sowie rund 90.000 Tonnen Flussbausteine bewegt werden. Erfreuliches Detail am Rande: Die erforderlichen Grundkäufe der rund acht Hektar Grund sowohl auf Völser als auch auf Innsbrucker Seite konnten mit allen GrundeigentümerInnen im besten Einvernehmen abgeschlossen werden.

\section{Muelheim Water Award 2008}

\section{Auslobung im Januar 2008 gestartet}

er Muelheim Water Award geht in die zweite Runde: Bewerbungen zum Thema „Fortschritte in der Wasserversorgung und der Abwasserentsorgung“ können seit Januar 2008 eingereicht werden. Der Bewerbungsfrist endet am 30. April 2008, $12.00 \mathrm{Uhr}$ MEZ.

Der Muelheim Water Award zeichnet herausragende Projekte zur praxisorientierten Forschung und zur Implementierung innovativer Konzepte zur Verbesserung der wasser- und abwasserwirtschaftlichen Situation aus, welche den Themenfeldern Ressourcen, Technologie, Qualität und/oder Organisation zugeordnet sind. Er wird alle zwei Jahre vergeben.
Das Preisgeld in Höhe von EUR 20.000 wird von der RWE Aqua GmbH gestiftet. Der Muelheim Water Award richtet sich an nationale und internationale Bewerber in und aus folgenden Zielländern: Belgien, Bulgarien, Deutschland, Frankreich, Italien, Kroatien, Luxemburg, Montenegro, Niederlande, Österreich, Polen, Rumänien, Schweiz, Serbien, Slowakei, Slowenien, Tschechien und Ungarn. Damit wurde die Zielregion auf insgesamt 18 Länder Zentraleuropas erweitert.

Im ersten Auslobungsjahr 2006 hatte die international besetzte Jury eine innovative Abwasserentsorgungslösung mit dem Thema „Dezentrale Behandlung und Wiederverwendung von häus- lichem Schmutzwasser in alpinen Gebieten“ als Gewinnerprojekt festgelegt. Als Preisträger wurde die terraLink $\mathrm{GmbH}$ aus Zürich ausgezeichnet. Das prämierte Projekt wurde gemeinsam mit dem öffentlich-rechtlichen Forschungsinstitut EAWAG (Eidgenössische Anstalt für Wasserversorgung, Abwasserreinigung und Gewässerschutz) durchgeführt.

Die Durchführung und Organisation des Preises erfolgt im Auftrag der Träger RWE Aqua GmbH und RWW RheinischWestfälische Wasserwerksgesellschaft $\mathrm{mbH}$ durch das Koordinationsbüro im IWW Rheinisch-Westfälisches Institut für Wasserforschung gemeinnützige $\mathrm{GmbH}$ in Mülheim an der Ruhr, Deutschland.

Weitere Informationen: Koordinationsbüro des Muelheim Water Award, Tel.: +49 208 40303-0, Fax: +49 208 40303-82,info@muelheim-water-award.com, www.muelheim-water-award.com 


\section{IMPEL}

\section{EU-Projekt zu Deponien un- ter österreichischer Leitung}

(OTS) Bei der Umsetzung der EU-Deponierichtlinie gibt es in vielen Mitgliedstaaten erhebliche Defizite. Vielfach werden notwen-

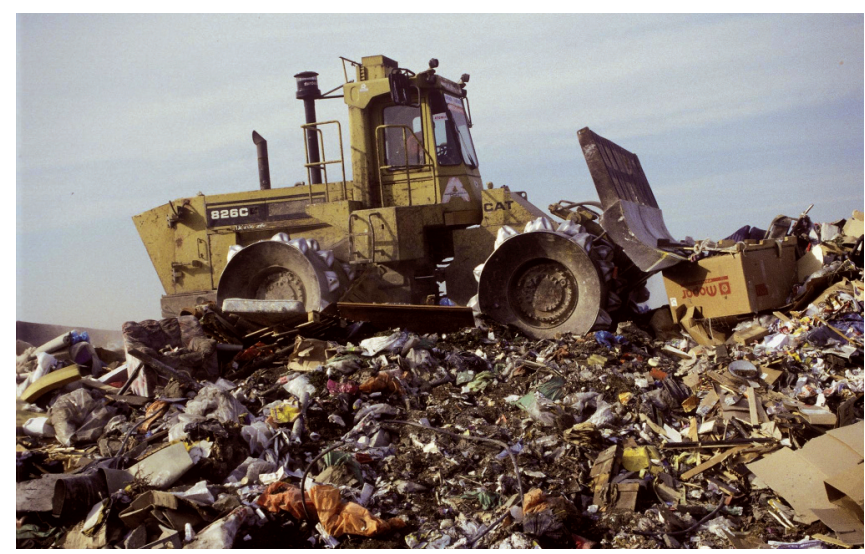

Deponie (Foto: BMLFUW/UBA/Gröger)

dige Schritte zur Umsetzung nicht vollzogen. Um eine einheitliche Anwendung und Durchsetzung der EU-Richtlinien in allen Mitgliedstaaten zu erreichen, hat die EU-Kommission das IMPEL-DeponieProjekt „Comparison Program on Landfill Inspection and Monitoring" ins Leben gerufen. Abfall-Experte Franz Mochty aus dem Lebensministerium wurde von der Kommission als Projektleiter bestellt. „Das Angebot, die Projektleitung zu übernehmen, zeigt das große Vertrauen der Kommission in die bisher in Österreich im Abfallsektor geleistete Arbeit", freute sich Mochty. Das erste Treffen der Projektteilnehmer fand am 18. Jänner $2008 \mathrm{im}$ Lebensministerium in Wien statt.

Das dem Deponie-Projekt übergeordnete EU-Abfallnetzwerk IMPEL (European Union Network for the Implementation and Enforcement of Environmental Law) setzt auf Erfahrungsaustausch und grenzüberschreitende Zusammenar- beit der EU-Länder. Österreich kann in diesem Bereich mit einem beachtlichen Know-how aufwarten. In der Jänner-Sitzung ging es vor allem um die Abstimmung der notwendigen Schritte bei der Abfallbeurteilung für die Deponierung, erfolgreiche Maßnah-
Enjoy Water" gestartet. Die neue Initiative zielt darauf $a b$, die Jugendlichen an den bewussten Umgang mit Wasser heranzuführen. Für Umweltminister Josef Pröll ist die Beteiligung von Unternehmen an der Jugendplattform „Generation Blue“ ein wichtiges Signal. „Enjoy Water“ wird bis Mai 2008 laufen.

Herzstück des Schulwettbewerbes ist der speziell für SchülerInnen gestaltete „Wasserpass“. Er enthält einfache Fragen zum Duschverhalten der Jugendlichen und bietet die Möglichkeit zur Aufzeichnung ihres Trinkverhaltens. So lernen die SchülerInnen spielerisch den bewussten Umgang mit Wasser als wichtigstes Gebrauchsgut und Lebensmittel. Darüber hinaus wird auf der Generation Blue-Homepage (www.generationblue.at) der GROHE-,,Wassersparrechner“ zu finden sein. Die SchülerInnen erarbeiten sich durch das Ausfüllen ihres Wasserpasses viele Informationen rund ums Wasser und bekommen so auch Antworten auf Fragen wie etwa „Wusstest du, dass du ca. 135 Liter Trinkwasser am Tag verbrauchst?" Auch die Kreativität der SchülerInnen ist beim Wettbewerb gefragt. Aufgabe ist es, einen originellen Spruch zu kreieren, der das Duschverhalten be-
Österreich den Schulwettbewerb „Mein Wasserpass - schreibt. Eine Jury ermittelt aus allen eingesandten Wasserpässen die 10 besten Sprüche. Die „Top Ten“ werden dann auf T-Shirts gedruckt und an den Gewinnerschulen gratis verteilt.

Um auch im regulären Unterricht für Abwechslung und für die Auseinandersetzung mit dem bewussten Gebrauch des kühlen Nass zu sorgen, stellen Generation Blue und GROHE zudem Unterrichtsmaterialien zur Verfügung. LehrerInnen können diese unter office@aqa.at gratis anfordern oder auf www.generation blue.at herunterladen.

\section{NOTWASSERVERSORGUNG}

\section{Gemeinsame Investition} von Wien und Klosterneuburg

(RK) Seit dem Jahr 1956 garantieren die Wiener Wasserwerke die Notversorgung der Nachbarstadt Klosterneuburg mit Trinkwasser, die im Schnitt an einigen Tagen pro Jahr - etwa bei Hochwasser oder bei anhaltenden Hitzeperioden - in Anspruch genommen wird. Seit dem Jahr 2005 wurden die dafür nötigen Anlagen erneuert und erheblich verstärkt. Die Inbetriebnahme der erneuerten Notwasserversorgung erfolgte am 6. Dezember 2007 im Pumpwerk Nußdorf. Mit dem Projekt wurde eine zusätzliche Versorgungslinie geschaffen, die die Versorgungssicherheit der AnrainerInnen an der Heiligenstädter Straße, im Kahlenbergerdorf, in der Kuchelauer Hafenstraße und in der Wiener Schüttau weiter steigert. Gleichzeitig können nun die BürgerInnen Klosterneuburgs in Notzeiten aus Wien mit bis zu 6 Millionen Litern Trinkwasser täglich versorgt werden. Zum Vergleich: Die beiden Wiener Hochquell- 


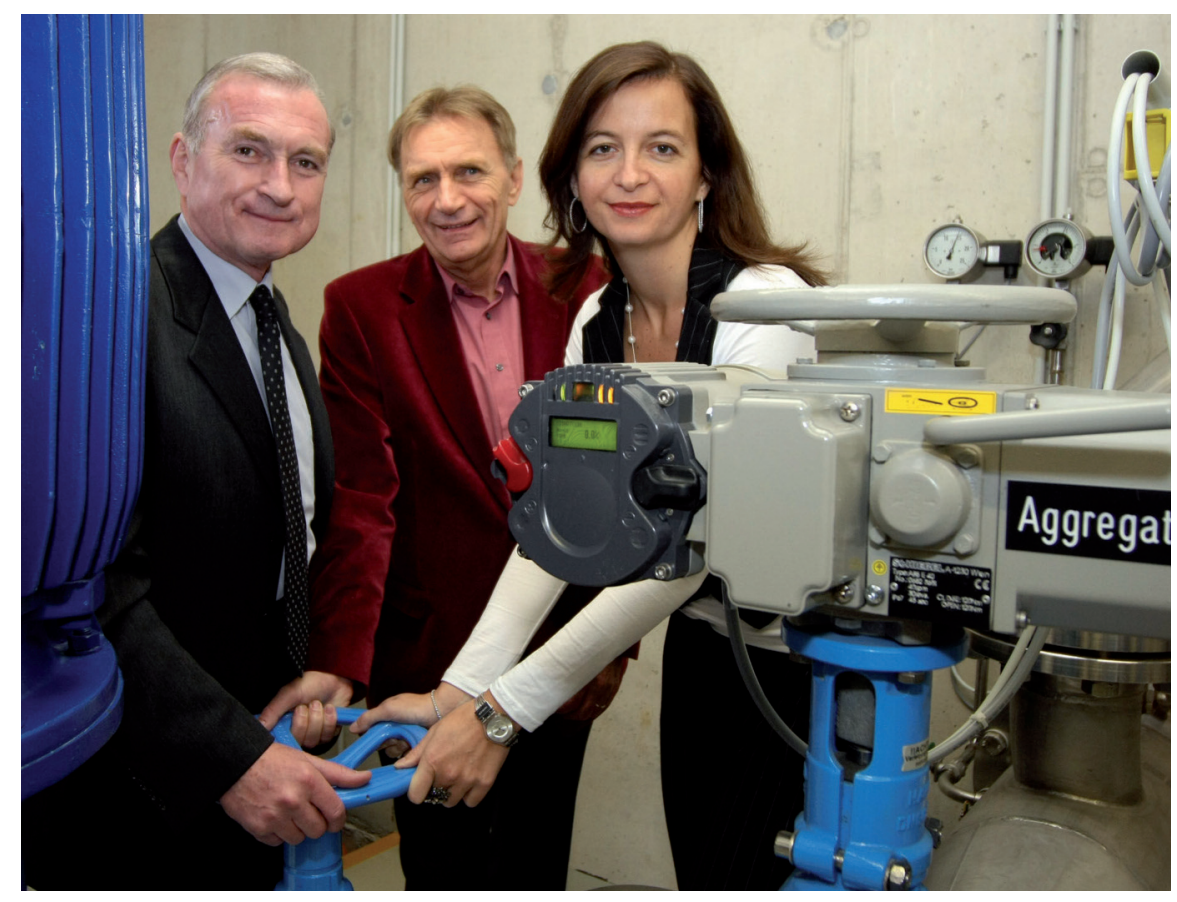

(v.l.n.r.): Bgm. Dr. Gottfried Schuh (Klosterneuburg), OSR DI Hans Sailer (Wiener Wasserwerke) und StR Mag. Ulli Sima bei der Inbetriebnahme der Notwasserversorgung (Foto: Pressefoto Votava)

leitungen liefern täglich bis zu 437 Millionen Liter Trinkwasser bester Qualität aus den niederösterreichischsteirischen Kalkalpen in die Bundeshauptstadt.

Neben der Erneuerung bestehender Trinkwasserleitungen umfasste das nun abgeschlossene Projekt die Verbindung der beiden Leitungen in der Heiligenstädter Straße im Bypass-Verfahren. Ein neuer Transportstrang entstand ausgehend vom Nußdorfer Platz über die ÖBB- und Schleusenbrücke bis zum Pumpwerk Nußdorf in WienBrigittenau. Um die größeren Wassermengen mit höherem Druck an die Stadtgrenze liefern zu können, war auch eine Erweiterung dieses Pumpwerks erforderlich. Die Gesamtinvestitionen für das Projekt belaufen sich auf 3,4 Millionen Euro, davon werden 1,4 Millionen Euro von der Stadtgemeinde Klosterneuburg getragen. Alle Anlagen stehen im Eigentum der Stadt Wien.

\section{UMWELTSCHUTZ}

\section{Stopp Littering - Großes \\ Engagement für ein sauberes NÖ}

Die niederösterreichischen Abfallverbände und das Land NÖ können dank der tatkräftigen Unterstützung von
Gemeinden, Veranstaltern und der Bevölkerung eine stolze Bilanz ziehen: Die Initiative „Stopp Littering - Wir halten Niederösterreich sauber!" erfreut sich eines regen Zuspruchs und großer Beteiligung. Mit der 2007 neu gestarteten Aktion „Sauberhafte Feste“ setzt NÖ einen weiteren Schritt in der Abfallvermeidung und macht Feste zu einem noch angenehmeren Erlebnis für alle. Im Rahmen einer festlichen Bilanzveranstaltung am 29. November 2007 in Tulln wurde die erfolgreiche Zusammenarbeit in Anwesenheit von Umweltlandesrat Josef Plank, LAbg. Alfred Riedl, Obmann des Abfallverbandes Tulln, und Alfred Weidlich, Präsident des NÖ Abfallwirtschaftsvereines, gebührend gefeiert. 2006 war der „Frühjahrsputz“ die erste Maßnahme der Stopp-Littering-Kampagne. Diese Flurreinigungsaktionen sind in vielen Gemeinden ein Fixpunkt der lokalen Umweltschutzagenda. In den ersten beiden Jahren der Kampagne wurden 815 Einzelaktionen durchgeführt. Dank dem Einsatz von 45.000 freiwilligen HelferInnen konnten 789 Tonnen Abfall aller Art gesammelt und ordnungsgemäß entsorgt werden. Die Aktionen sollen auch im Frühjahr 2008 fortgesetzt werden.

Die „Sauberhafte Feste“-Qualität hat sich in Niederösterreich bereits herumgesprochen. Seit dem Start der 


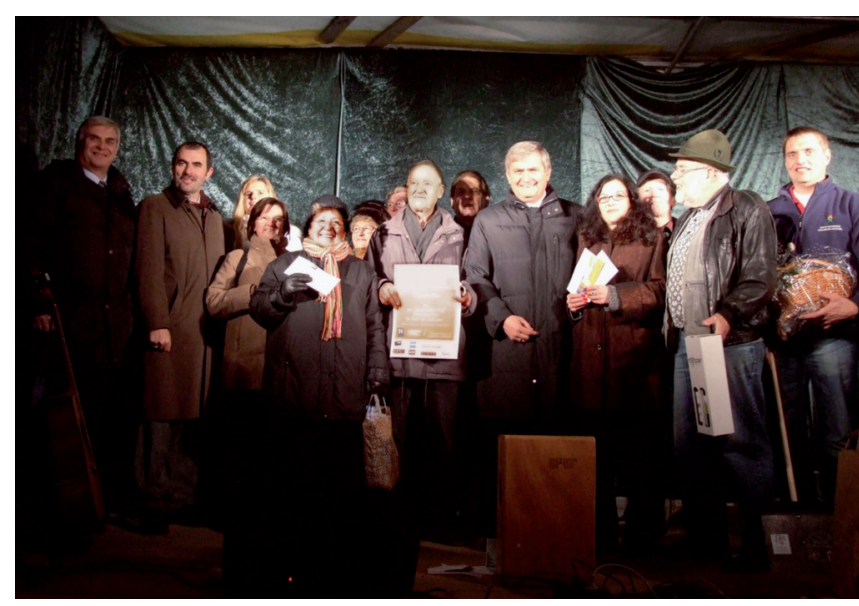

LR DI Josef Plank, Präs. Dipl.-Päd. Alfred Weidlich (NÖ AWV) und LAbg Mag. Alfred Riedl bei der Bilanzveranstaltung von „Stopp Littering“ in Tulln (Foto: NÖ AWV)
Aktion im Mai 2007 bis heute waren insgesamt 344 Festtermine auf der zentralen Informations- und Serviceplattform www.sauberhafte feste.at registriert. Sie alle vom kleinen Grätzelfest über Feste von Feuerwehren und Sportvereinen bis zu Großveranstaltungen wurden ohne Müllberge gefeiert und anschließend wurde für die optimale Trennung der Abfälle geMehrweggeschirr und Trennung aller Festabfälle lassen sich die Rest-Abfallmengen pro Person auf bis zu 0,25 Liter reduzieren! Diese umweltfreundliche Art der niederösterreichischen Festkultur wird mit dem Gütesiegel „Sauberhafte Feste“ ausgezeichsorgt - durch Einsatz von
OTTI in der Schweiz an: Die im November 2008 in Kraft tretenden kostendeckenden Vergütungen für Wasserkraftwerke bis 10 Megawatt mittlerer Bruttoleistung haben zusammen mit den gestiegenen Marktpreisen eine gewaltige Nachfrage nach Wasserkraftwerken ausgelöst.

Zwanzig Jahre, nachdem eine Expertengruppe des Bundes aufgezeigt hat, dass ein Potenzial zur Verdoppelung der Produktion aus Kleinwasserkraftwerken besteht, macht sich nun die Schweiz daran, einen guten Teil davon systematisch zu erschließen. Das Anwenderforum leistet dazu einen kleinen Beitrag und kann Impulse in die Nachbarländer bringen.

Themenschwerpunkte des Anwenderforums werden sein:

- Planung

- Technik

- Betrieb

- Umwelt

- Wirtschaftlichkeit

- Rechtliche Fragen

\section{KLEINWASSERKRAFT}

\section{Internationales Anwen-} derforum 2008 in Luzern

(OTTI) Das 11. Internationale Anwenderforum Kleinwasserkraftwerke (25.26. September 2008, Luzern) bietet sich ideal als erste Veranstaltung des

\section{BAUTECHNIK}

\section{Generationswechsel an der Spitze der MA 39}

(RK) DI Georg Pommer (46) ist der neue Leiter der PrüfÜberwachungs- und Zerti-
Weitere Informationen: www.otti.de

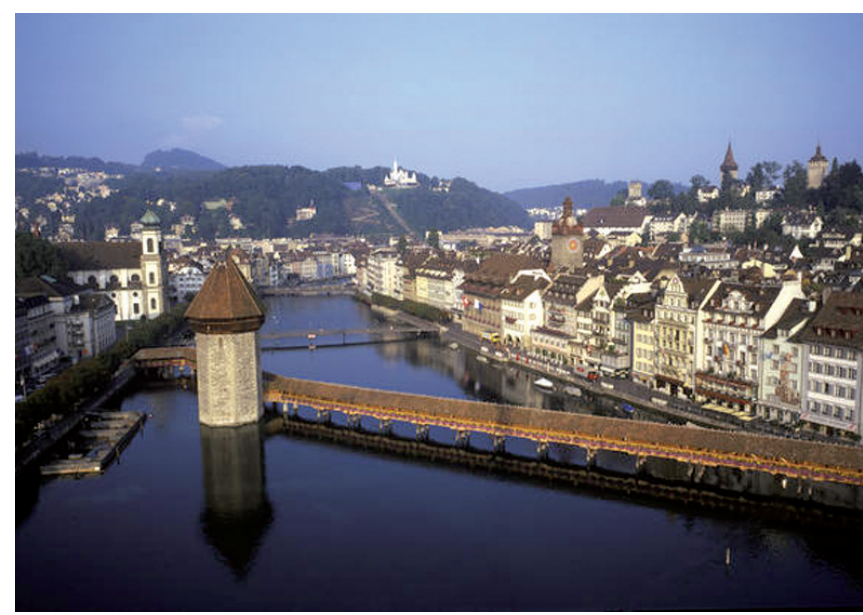

Die Kapellbrücke in Luzern (Foto: Luzern Tourismus \& AURA) fizierungsstelle der Stadt Wien (Magistratsabteilung 39). Er war zuvor Stellvertreter des bisherigen Chefs der Abteilung, DI Werner Fleck (64), der in den Ruhestand getreten ist. Magistratsdirektor Dr. Ernst Theimer überreichte dem neuen Abteilungsleiter am 9. Jänner 2008 in Anwesenheit von Wohnbaustadtrat Dr. Michael Ludwig, zu dessen Ressort die MA 39 gehört, sein Bestellungsdekret.

Die Prüf-, Überwachungsund Zertifizierungsstelle der Stadt Wien ist die älteste bautechnische Prüfanstalt Österreichs. Gegründet im Jahr 1879 ist sie heute als akkredidierte Prüf- und Überwachungsstelle tätig und in der Europäischen Union notifiziert. Mit dem Ziel der Weiterentwicklung zu einem KompeMA 39 kürzlich auch die Aufgaben des Instituts für Umweltmedizin und der physikalisch-technischen Prüfanstalt für Strahlenschutz übertragen. Der neue Chef Georg Pommer studierte an der Universität für Bodenkultur Holzwirtschaft und arbeitet seit 1986 in der MA 39. 1992 wurde er Leiter des heutigen Hochbaulabors und Personalreferent. Seit 1996 war er Stellvertreter des Abteilungsleiters. tenzzentrum wurden der

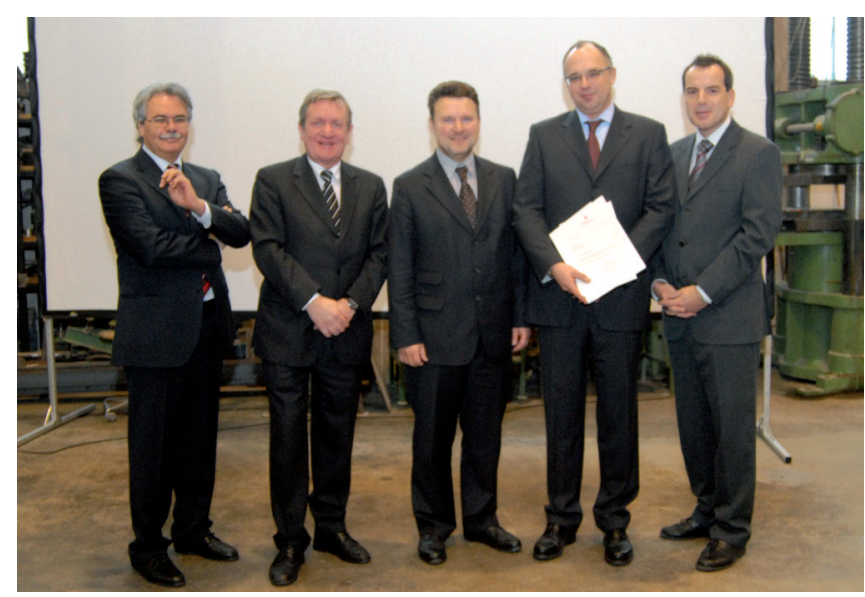

(v.l.n.r.): DI Wilhelm Wimmer (Stadtbaudirektion), MD Dr. Ernst Theimer, StR Dr. Michael Ludwig, DI Georg Pommer, Christian Meidlinger (Foto: media wien) 